Gowers has placed the specific cases at 80 per cent. and only accounts for 10 per cent. of idiopathic, possibly we can say locomotor ataxia without specifying the pathology.

I have a case of specific trouble in which this analgesia is present. The pationt has tactile sense but loss of feeling in patches.

Dr. HAROLD N. Moyer-Since the publication of the papers referred to 1 have examined seven cases and the symptoms were present in all. These are the only cases I have examined, with the exception of two chldren, one a very marked ataxic; both cases of Friedreich's disease; neither of these children has any of the zones of analgesia or anesthesia. I have observed it, however, in one case of well marked syphilis in the spinal cord, which would not be pronounced in any way an ordinary case of tabes; it was upon one side, and corresponded to a very marked paretic condition on that side.

Dr. Charles W. Burr of Philadelphia - I have recently examined two cases of Friedreich's ataxia, and there was no such trunk anesthesia present.

\section{THE USE AND ABUSE OF ELECTRICITY IN THE TREATMENT OF THE SO-CALLED NEUROSES.}

Presented to the Section on Neurology and Medical Jurisprudence at the Forty-eighth Annual Meeting of the American Medical Association, beld at Philadelphia, Pa., June 1-4, 1897.

BY L. HARRISON METTLER, A.M., M.D. CHICÁGO, YLL.

Of all the therapeutic measures employed to com bat disease, electricity is probably the most abused This is due partly to ignorance, partly to indifference. The extreme diversity of opinion in regard to it as an agent for good is ample testimony of the widespread ignorance concerning its powers. It is a fact that electricity is beneficial in the treatment of diserse or it is not; it can not possess two dissimilar qualities at one and the same time. It is absolutely inconceivable that electricity should be at once perfectly neutral and most curative under similar conditions. Therefore, the differences of opinion held in regard to it can not be attributed to anything in the agent itself. The differences of opinion, must have their source in those who use the agent. The users themselves must differ either in their knowledge or in their methods of handling it. These assertions, I admit, are trite, but if their truth can be brought home to those who attempt to employ electricity something may be gained in the way of more extensive positive knowledge and greater uniformity of opinion.

When one operator insists that all strictures can be reduced by electricity, and another says no strictures can be so affected, surely the electricity can not be at fault for that is presumably the same in both hands. The discrepancy lies with the operators. When Möbius of Berlin argues that the only positive effect of electricity upon a patient is of a mental, suggestive or psychic sort, and Apostoli of Paris teaches that solid fibromata may be made to vanish under the solvent action of the electric current, it is time for us to enquire wherein the methods and personal characteristics of Möbius and Apostoli differ; for, presumably, they have both used the same electric current-the only electricity known in this world - upon the same sort of pathologic conditions, and under corresponding circumstances. The question therefore becomes largely one of personal honesty and relative powers of interpretation. Many things appear different to different men, but if eletricity produces certain physiologic phenomena, such as muscular contraction, catalysis, cataphoresis and modification of local temperature, it is incomprehensible that these obvious phenomena should not be seen by optimist and pessimist alike. Surely, if muscles do really contract under electric stimulation, the patient as well as the operator can not honestly declare that such contraction is a sort of mental aberration or psychic ballucination. If chemic changes are really wrought in the fluids of the body by the poles of a battery, certainly the fact must be something more than a mere figment of the imagination.

It is universally admitted that electricity does produce certain manifestations in the normal tissues, these manifestations always being the same under the same conditions. I enumerate some of those physiologic actions upon which experimenters are pretty well in accord. They are to be noted as dynamic, electrotonic, thermic, electrolytic, catalytic and cataphoric. There are other effects less constant, and some, as Erb says, "that we may not now have even a suspicion of," but those I have mentioned are constant and apparent. As a mechanical irritant and as a chemic resolvent, electricity has undoubted force. Certain details and minutiæ in the manifestations of this force may at the present time be open to controversy, but that the electric current is not absolutely inert in its effect upon the body is admitted. It certainly does influence nerve currents, however that influence may be explained. Its electrotonic force diminishes or increases the transmission of sensory and motor impulses along the respective nerves, according to the ascending or descending direction of the electric current. Muscles do most obviously contract, whether the pole of the battery be applied directly to the muscular tissue or to the related nerves, and Pflüger's famous law in regard to these contractions is a positive scientific fact. Local anemia and hyperemia are, beyond dispute, caused by the action of the electric current. Chemic, mechanical and physiologic effects are most clearly discernible in the skin, as a result of the application of the galvanic current. The same may be said of its action upon the blood.

In addition to these particular activities, there is a whole series of manifestations produced by the dynamic effect of the direct application of the electric current to the brain, the spinal cord, the sympathetic nerves, and nerves of special sense, the sensory and motor nerves, the voluntary and involuntary muscles and the various glands and organs.

The functions and products of the secretory glands are modified by the irritative action of the electric current, sometimes being increased and sometimes diminished according to the kind and quantity of electricity used. Simple observation shows that the blood is affected by the current and that clots are formed while hydrogen and oxygen as well as acids and alkalies are evolved at one or the other electric needle immersed in the blood stream. The local effects upon nutrition are apparent enough to any unprejudiced observer and are to be noted as mechanical, physic, chemic and physiologic.

It is not my desire to refer more minutely to the great subject of electro-physiology. I wish merely to emphasize the fact that there are electro-physiologic phenomena, and to consider how these effects are to be utilized in the treatment of a special class of diseases.

Why is electricity as a therapeutic measure so misused? Many reasons might be suggested, but most of them would come under the head of ignorance or indifference. The expense of a complete electric out- 
fit and the trouble of applying the agent are two important hindrances to its universal adoption. It is easier and less expensive to write a prescription than to give an electric treatment. To tell a patient to purchase a "family" battery and to hold the electrodes in the hands while "the current is flowing," is about as sensible as to tell him to go to a drug store and take "some" strychnin as strychnin is a good "nerve tonic." Not to know when and how to employ the galvanic instead of the faradic current, reveals about as much knowledge of electro-therapeutics as not to know when and how to administer belladonna. Far too many practitioners do not distinguish between the various currents, galvanic, faradic and static, in their applications. It is all electricity, they loosely argue, and if the patient is not benefited by the form of electricity which has been adminnstered, they conclude the agent is of no value. Again, they do not measure the dosage of the electricity given, but too often depend upon the feelings of the patient. In some varieties of disease the electric current works deleteriously if it is so strong as to cause the patient to be conscious of it. It is a mystery, therefore, how in such cases the electricity is to be administered with any sort of intelligence, if no measure of the dosage is employed. I have occasionally known the practitioner to first try the current upon himself and depend upon his own sensibility as a guide to the administration of the agent to the patient. One might as well exhibit quinin or opium to oneself with no thought of human idiosyncracies or differences of constitution as a guide for the exhibition of the drug to the patient.

Human nature is full of prejudices and more than once have the slower results obtained by electricity in certain cases been frowned on because of the more brilliant and rapid effects produced by surgery. All prejudice aside, however, it is a fact that in many of the neurotic affections-especially the neuroseselectricity is the curative agent par excellence, and while there is less eclat associated with it than with a surgical operation, it accomplishes its results without mutilation. So careful and conservative a clinician as Erb of Heidelberg says that "electricity is an extremely powerful and many-sided remedy and that more evident and undoubted curative effects may be attributed to it in diseases of the nervous system than to almost any other remedy. I am not guilty of exaggeration when I say that the curative effects not infrequently astonish even the experienced physician by their magic rapidity and completeness."

And yet there are grave difficulties encountered sometimes in the treatment of these diseases with electricity. They are largely, however, diagnostic and pathologic. Everyone admits that, physiologically, electricity stimulates nutrition. Naturally it follows that in those troubles in which diminished nutrition is the principal factor, electricity should play an important rôle therapeutically. Here is where much of the differences of opinion in regard to the value of electricity have their origin. What are the nutritional diseases and how can they always be accurately diagnosed?

Again, it is recognized that physiologically, electricity manifests a certain amount of catalytic power. Most rational would it seem, therefore, to employ this agent in the treatment of those conditions, like tumors, in which a dissolution of the tissues and fluids is desirable. But who will always and positively tell what conditions are amenable to this solvent force? Obviously we have many things to learn, and it is undeniable that electricity, like a host of other therapeutic agents, must to a large extent be used empirically. It behooves us not to condemn a measure which empirically is shown to be beneficial merely because we do not fully understand the pathology of the diseases in which its grand effects are so apparent.

A neurosis or functional disease is one of the enigmas of medical science. All that we can say is that the tissues and organs involved are functionally perverted, which is nearly equivalent to saying nothing. Many of the so-called functional troubles are purely reflex in character. Sometimes we can discover the distant organic lesion that is the prime cause of the reflex disturbance; more often, however, this final cause remains undiscovered or is found only after the patient has been subjected to a number of mutilating surgical operations. And in very many cases the functional trouble remains after all supposed organic lesions have been removed. Who has not seen cases in which ablation of the ovaries for epilepsy or insanity has failed to accomplish the desired result? Not always does a circumcision put a stop to a chorea or the fitting of a pair of glasses remove a headache. It is well that these operative procedures should be done when needed to clear up strong presumptive evidence that the organic lesions are the source of the neurotic troubles. Other measures, however, should always be given a thorough trial before any such radical methods are instituted. The ultra-mechanical treatment of such conditions as dysmenorrhea, neuralgia, vaginismus, epilepsy, seminal incontinence, intestinal constriction, etc., is not always successful even when most radically undertaken. To argue that an operation is always radical is no argument against trying other measures first. Radicalism is not always effective and too often transcends the limits of what is judicious and necessary. There are men who propose the production of abortion as a radical cure for severe vomiting of pregnancy. By the same process of reasoning these men would probably declare for amputation of an arm for a painful neuroma. Medicine should conserve as much as possible, and for that reason heroic measures should always be approached gradually and only as a last resort. Since therapeutics is only a means to an end and not the end itself, the harsher the means the more reluctantly they should be adopted. If a neuralgia can by any possibility be alleviated by electricity, no argument on the ground of radicalism can console a patient for a surgical mutilation.

There is a large class of neuroses in which the cause of the trouble seems to be inherent in the tissues themselves. The best explanation we can offer of their pathology is that they are the result of impaired nutrition. The chemic or molecular state of the nerve cells is in some way at fault. Perchance this is brought about by a local vasomotor irregularity or by some inherent defect in the metabolic functions of the cells themselves. If the circulation is not what it ought to be, the pabulum carried by the blood to the nervous structures will be deficient or in excess, and these structures will be starved or overnourished. On the other hand, if the cells themselves are wanting in tone so that the pabulum is not normally assimilated and metamorphosed into their own chemic or molecular condition, these structures will not functionate as they should, but give rise to a so. 
called functional disease. There may be, and in some instances undoubtedly are, irritants and toxins in the blood that exert a deleterious influence upon the delicate nerve cells so as to modify their functional activities. Many cases of chorea are due to the uric acid diathesis, while not a few mental symptoms are believed to be caused by a toxic condition of the blood. Even in these cases, however, the essential trouble in the histologic structures is largely nutritional, whatever the nature of the prime cause may be. All this class of cases, the so-called neuroses, are therefore especially amenable to electric treatment. Hysteria, insomnia, spinal irritation and nervous exhaustion are always immensely benefited by the electric treatment when it is properly administered. When I see, as I have in many of these cases, an improvement in every symptom, an increase of body weight, a reawakening of the appetite and a more natural performance of all the bodily functions, I am at a loss to understand how such results can be attributed solely to the mental impression or psychic influence excited by the electric current. I can as easily believe that a brick wall may be raised by the practice of hypnotism upon it by the master mason as to believe that such physical manifestations as restored digestion, circulation and restored functions of all sorts in a neurasthenic are the result of a kind of electro-hypnotic influence. I would have to convince myself that electricity does not really stimulate, does not irritate, that in fact it is physically absolutely neutral in its effects, local and general, while the patient's mind alone brings about all the wonder. ful changes in the activities and appearances of the bodily tissues. No, the psychic theory in regard to the power of the electric current is not only irrational and illogical, but it is emphatically opposed to most observable and tangible facts. I am not over-enthusiastic when I say that in the treatment of the neu. roses electricity is the sovereign remedy. In selected cases it accomplishes most favorable results, as I could easily show from my case-book. Whether the influence of the electric current upon the nutrition be the sole explanation of its modus operandi or whether in addition to its electrotonic, catalytic and cataphoric properties, it possesses other more subtle powers, is a matter of small consequence in discussing the reality of its effects in the treatment of the neuroses. Because it is so powerful an agent is the very reason why it provokes such a diversity of opinions. Electricity is not a panacea even in the treatment of the neuroses, its most favorable field. In accordance with our present knowledge only that class of cases in which nutrition is primarily at fault call for it. $\mathrm{As} \mathrm{I}$ have already indicated, certain nenroses are mere reflex manifestations and while electricity may benefit them for a time, it can not be expected to produce a cure until the original cause of the reflex is removed.

A young woman was recently referred to me who had been treated for three months by a gynecologist, on the theory that her neurotic symptoms, which were most distressing, might be due to some pelvic disease. Showing no improvement as a result of the local treatment, she took electricity, baths, massage, etc., and persisted in the general treatment greatly to her benefit. She was an orphan, of a naturally sensitive nature, overwhelmed with financial troubles in connection with her estate, and uncertain as to the future of her life. All these factors had combined to institute a train of neurotic manifestations such as insom- nia, restlessness, irritability, loss of appetite, nervous dyspepsia, weakness of memory, want of the power of concentration, fugitive pains, backache, etc. Exam. ination of the pelvic organs, by myself as well as by others, discovered no pelvic trouble whatever. I was surprised that the simple history of this case did not have more influence in the diagnosis made by the gynecologist, but when I found no disease whatever of the pelvic organs, I naturally had my suspicions in regard to the honesty or ability of her attendant. Another case sent to me was that of a young woman who had received much electric and anti.hysteric treatment near her home, without apparent benefit. She was herself to blame in this instance, in having been unwilling to submit to a vaginal examination. This examination, made by myself and by another, on account of her persistent backache, leucorrhea and dysmenorrhea all coming on shortly after a severe fall, revealed a pronounced retroflexion of the uterus with chronic endometritis. In such a case electricity was naturally without benefit to the neurotic condition except temporarily. Three months' treatment here for the pelvic trouble sent the girl home well, happy and grateful.

I have related these two well marked contrasting cases to illustrate the use and abuse of electricity. I could give others, even more in detail but mere repetitions consume time without adding information. A correct diagnosis is an absolute sine qua non in the proper administration of electricity. Too often, however, it seems that with some practitioners the more obscure the diagnosis is, the greater the need for electric treatment. If a paralysis or a neuralgia, for instance, can not be assigned to a definite cause, then as a last resort electricity is tried. I think I am not far out of the way in saying that when a set of neurotic symptoms remains unrelieved by the application of the electric current, the practitioner, to be honest to himself and to his patient, should conclude that his diagnosis is wrong and that his therapeusis is being misapplied. The supposed neurosis is in all probability a reflex of some sort, and calls for other treatment than the electric. More than once have I had headache cases come to me in the hope of being benefited by electricity, while a most superficial examination indicated some form of eye strain. I must frankly state, however, that I have had not a few cases which had been fitted with glasses, return to me with no modification of the old headache, until a more thorough course of general and electric treatment had been instituted. Neurologists are acquainted with such a condition as pain habit, and in lieu of a better explanation I find I have to attribute some of these pains to such a habit.

In the electrical treatment of the neuroses, I find that a combination of galvanism and faradism gives the happiest results. I use a galvano-faradic apparatus. In galvanization of the spine and general muscular faradization, I have the patient remove all clothing and lie on a couch, without exposure, under a large sheet. Sponge electrodes about five inches in diameter, having convenient handles attached are used. At first, with the small, ordinary hand electrodes, one at the nape of the neck, the other over the sacrum or epigastric region, the spine and sympathetio system of nerves are galvanized for four or five minutes. The feeblest possible current is used, the strength never being greater than is just sufficient to cause a slight metallic taste in the mouth. By measurement 
it should not be more than from five to fifteen milliamperes. At this stage of the treatment the patient usually declares that he feels a decided local warmth at the negative pole. While maintaining the electrodes in their respective positions, the current is reversed some five or six times with the current reverser of the battery.

With the larger sponge electrodes general faradism is applied to every part of the body. One pole is placed at the nape of the neck or under the sacrum, while the other is passed successively over all the muscles causing in them a gentle but decided contraction. Many patients are over-sensitive, and in such it is advantageous for the physician to hold one pole of the battery in his left hand while he uses his right hand to administer the electricity with a gentle massage to the patient. This is more tiresome to the physician, and fortunately is necessary in only a small percentage of cases. I have used the method many times without feeling any bad effects from the electricity passing through myself. The advantages are that the physician can control the strength of the current better and a sensitive, nervous patient is not annoyed by any sudden shocks. While the spinal galvanism should not be prolonged beyond five minutes, as a rule, the general faradism is gradually increased in strength and time until the latter is an hour and the former produces almost painful contractions of the muscles.

In selected cases this simple method of administering electricity affords, after a few seances, a grateful feeling of rest, sleepinese and well being, which continues until in the end the whole constitution partakes of the improvement, and the patient looks and feels once more like a normal being. If after the firat two or three applications a restful feeling and a desire for sleep are not experienced, the physician's suspicions should be awakened. If a condition of over-excitability is produced he may be quite certain that his diagnosis is wrong, and the case is one not amenable to electric treatment.

With a proper knowledge of the qualities of the electric current, with more care in the making of the diagnoses and the selection of cases, and with an intel. ligent scientific application of the agent, electricity, in the treatment of the neuroses ought not to be abused as much as it is, but it ought to be used with a degree of success that will award it its due place among our therapeutic resources as one of the most powerful and effective in the conflict with disease.

4544 Lake Ave.

\section{A CASE OF LOSS OF BLOOD FROM THE RECTUM DUE TO MUCO-MEMBRANOUS ENTERO-COLITIS, AND TWO OF LEUKEMIA.}

Clinic delivered at the Jefferson Medical College Hospital. BY H. A. HARE, M.D.

Professor of Therapeutics and Materia Medica, Jefferson Medical College of Philadelphia.

PHILADELPHIA, PA.

The first patient is a man of 23 who is somewhat thin and anemic, and whose face shows the signs of mental depression and lack of nervous energy. His history is, that he was in perfect health until about two years ago, when he was suddenly seized with a severe hemorrhage from the bowels, the blood being quite bright in color. He tells us, however, that he has had no diarrhea at any time, but on the contrary suffers from obstinate constipation. There is nothing in his history which points clearly to the cause of these bloody movements, which have continued from time to time, generally once or twice a week ever since he was first taken ill. The frequent loss of blood has resulted in general debility and anemia. He tells us that an examination of his passages not only reveals blood but a large amount of mucus which appears in shreds or stringy masses. He often has much griping pain and flatulent distension, his appetite is variable and his diet is an irregular one because he is afraid to eat certain articles lest they produce an increase in his discomfort. Every few days he is seized with an attack of looseness of the bowels which seems as if it were an effort upon the part of the bowel to get rid of an excessive quantity of mucus and feces which have accumulated. His urine is normal.

When a case of hemorrhage from the bowels comes before you, the possibility of it arising from a number of different causes at once enters your mind. First, it is possible and most probable in such a case that the hemorrhages come from hemorrhoids, but the man tells us that he has no sign of any congestion of his hemorrhoidal veins. This does not, however, prove positively that the blood has not this origin, for the most severe cases of bleeding from the rectum are seen in those persons who have no large dilatations of the hemorrhoidal veins, but in their place have small arterial twigs in a dilated and hyperemic state and these little bunches of vessels containing arterial blood, when scraped or eroded by fecal matter give free vent to little arterial spurtings. A careless examination of the rectum might result in their being overlooked, but a careful examination with a speculum will often reveal their presence.

Relief may be obtained by touching them very lightly with the tip of a fine glass rod dipped in nitric acid, the resulting scar puckering the blood vessels and preventing further hemorrhage, or in other cases an application of nitrate of silver or of dilute nitric acid for its astringent effect does equally well. I have had this young man carefully examined, however, and the report is that there is no sign of such bleeding points and we have therefore to look further for the cause of his trouble. Is it possible that the blood arises from a duodenal ulcer? I think not; for first, duodenal ulcer is a rather rare condition, and second, the blood which comes from such an ulcer is nearly always dark and tarry, through the action of the digestive juices of the bowel upon it, whereas the blood this man passes, he tells us, is quite bright and arterial in hue. Then, too, he has no pain in the neighborhood of the duodenum; such as is usually felt when ulcer of this portion of the alimentary canal is present and, finally, the blood is not well mixed with the fecal matter, but is well separated from it in the movements, although if it arose in the small intestine we would expect it to be thoroughly mixed with the stools. On the other hand, duodenal ulcer is far more frequent in males than in females. I think for the reasons given we may exclude the possibility of the blood arising from an ulcer in the duodenum.

The next cause to be thought of is cirrhosis of the liver. This condition, as is shown so well in the illustration of the results of cirrhosis of the liver in my book on "Diagnosis," results quite frequently in grave engorgement of the various abdominal viscera, 